\title{
The role of computed tomography and magnetic resonance imaging in diagnosing clear cell ameloblastoma: A case report
}

\author{
SHOKO GAMOH $^{1}$, MASAHIRO WATO ${ }^{2}$, HIRONORI AKIYAMA ${ }^{1}$, KANAME TSUJI $^{3}$, HIROKI ISHIKAWA ${ }^{3}$, \\ KAORI NARUSE ${ }^{3}$, HIROAKI YOSHIDA ${ }^{3}$, SHOSUKE MORITA $^{3}$, AKIO TANAKA ${ }^{2}$ and KIMISHIGE SHIMIZUTANI ${ }^{1}$ \\ ${ }^{1}$ Department of Oral Radiology, Osaka Dental University, Osaka 540-0008; ${ }^{2}$ Department \\ of Oral Pathology, Osaka Dental University, Osaka 573-1121; ${ }^{3}$ First Department of Oral \\ and Maxillofacial Surgery, Osaka Dental University, Osaka 540-0008, Japan
}

Received April 9, 2016; Accepted June 21, 2017

DOI: $10.3892 / \mathrm{ol} .2017 .7116$

\begin{abstract}
Ameloblastoma is the most common and clinically relevant type of odontogenic tumor. Clear cell odontogenic carcinoma is histologically characterized by solid sheets and nests of clear cells, whereas clear cell ameloblastoma (CCAM) is histologically characterized by an ameloblastomatous component intermixed with an extensive clear cell component. A total of 12 reports have been published on the histological etiology for CCAM; however, no reports have made regarding the detailed computed tomography and/or magnetic resonance imaging features of tumors of this type. The present study describes a case of a well-circumscribed 20-mm radiolucent lesion of the anterior mandible that was misdiagnosed as a clear cell odontogenic carcinoma. The study describes the detailed radiological characteristics of a case of CCAM.
\end{abstract}

\section{Introduction}

The ameloblastoma is the most common and clinically relevant odontogenic tumor (1). Our institution, Osaka Dental University (Osaka, Japan) treated 111 cases of biopsy-proven ameloblastoma from 2005-2014. Ameloblastoma is typically benign while locally invasive, with a considerable tendency to recur when not adequately removed (2).

In 1986, Muller and Slootweg (3) observed that clear cell differentiation may occur as a histological feature of ameloblastoma; the clear cells contain glycogen-rich cytoplasm. Mari et al (4) stated that the clear cell appearance in ameloblastoma could be considered as a new histological variant (4). Waldron et al (5) reported 2 cases of a lesion that

Correspondence to: Dr Shoko Gamoh, Department of Oral Radiology, Osaka Dental University, 1-5-17 Otemae, Chuo, Osaka 540-0008, Japan

E-mail: gamo-s@cc.osaka-dent.ac.jp

Key words: clear cell ameloblastoma, ameloblastoma, clear cell odontogenic carcinoma, dental radiograph, computed tomography, magnetic resonance imaging, radiological character they designated as 'clear-cell ameloblastoma' or CCAM, an odontogenic carcinoma. The 2 cases demonstrated an unusual biphasic pattern, with areas of typical follicular ameloblastoma together with a conspicuous clear-cell component. The clinical course indicated that these lesions should be considered as low-grade odontogenic carcinomas. Braunshtein et al (6) reviewed the reported features of clear cell odontogenic carcinoma and CCAM and suggested that these two types represented a continuum of the same neoplasm. Thus, CCAM is well-documented in the English-language literature; however, it is not officially recognized as a neoplasm by the World Health Organization.

To the best of our knowledge, details of the features of CCAM identified from cross-sectional imaging modalities, including computed tomography (CT) and magnetic resonance (MR) imaging, are unclear at present, due to the rarity of the disease and the past underdevelopment of these imaging modalities. Therefore, the present study reports a solid tumor in the mandible of a 40 -year-old male, which exhibited features that were notably similar to a desmoplastic ameloblastoma on CT and MR imaging. The unique process of reaching the correct diagnosis is presented, with a brief review of the literature on this topic.

\section{Case report}

A 40-year-old Japanese male was referred to Osaka Dental University by his dentist in 2015 for the inspection of a swelling in the area of the right canine of the mandible (Fig. 1). The patient had noticed the swelling for 4 months. An initial clinical examination presented a localized, elastic, hard swelling in the right buccal vestibule around teeth 42-44. The overlying mucosa was normal. An electric pulp test was performed due to the newly onset tenderness associated with the tooth, and the pulp was observed to be vital. An initial intraoral $x$-ray demonstrated a radiolucent lesion with delicate septa and margins near the apexes of teeth 42,43 and 44, mildly dislocating 42 and 43 (Fig. 2). An occlusal x-ray revealed a radiolucent-radiopaque lesion with a buccal bony expansion in the right lateral incisor and canine area of the mandible, illustrating an indistinct soap bubble-like appearance (Fig. 3). A panoramic $x$-ray image detected nothing relevant to the 
primary complaint of the patient and was affected by ghost images from the cervical vertebrae (Fig. 4). CT images were obtained using a CT scanner (BrightSpeed Elite; GE Healthcare, Chicago, IL, USA) at $120 \mathrm{kV}$. The electrical current was automatically optimized for the object thickness (maximum, $120 \mathrm{~mA}$ ). In addition, $\mathrm{CT}$ imaging was performed with the following parameters: Slice thickness, $0.65 \mathrm{~mm}$; pitch and tube voltage, $0.625: 1$; and field of view, $16.8 \mathrm{~cm}^{2}$. CT imaging revealed an expanding, mixed radiolucent-radiopaque appearance with poorly defined borders, including irregularly thinned cortical plates (Fig. 5A-C). The mass was a multiloculated, honeycomb-like lytic lesion containing a high number of septa. The CT value of the radiolucency inside the lesion was 30 Hounsfield units (HU), indicating fluid, and those of the septa were $\sim 120 \mathrm{HU}$, suggesting that there was an extent of calcification. Teeth 42 and 43 were slightly displaced, without root resorption. Neither a destructive condition nor inflammatory osteosclerosis were observed. MR examination was performed using a $1.5-\mathrm{T}$ superconducting magnet (SIGNA $^{\mathrm{TM}}$; GE Healthcare) with an 8-channel head and neck coil. T1-weighted spin-echo sequences [repetition time $\left(\mathrm{T}_{\mathrm{R}}\right) /$ echo time $\left(\mathrm{T}_{\mathrm{E}}\right)=760-800 / 8$-10], $\mathrm{T} 2$-weighted fast spin-echo sequences $\left(T_{R} / T_{E}=5,000-5,250 / 100-200\right)$ and $T 2$-weighted fast spin-echo sequences with the fat-suppression technique of the chemical shift selective method $\left(\mathrm{T}_{\mathrm{R}} / \mathrm{T}_{\mathrm{E}}=2,500-3,000 / 80-120\right)$ were obtained with a $20.0 \times 20.0 \mathrm{~cm}$ field of view and slice thickness of $4 \mathrm{~mm}$, with $1 \mathrm{~mm}$ spacing. Axial T1-weighted images (T1WI), axial T2-weighted images (T2WI), axial T2-weighted fat-suppressed images (T2WI-fat), coronal T1WI, and coronal T2WI were obtained. MR imaging revealed nonhomogeneous mixed signal intensities in the equivalent region. In T1WI it demonstrated an intermediate signal intensity (Fig. 6A), whereas in T2WI-fat it exhibited variable intermediate and high signal intensities (Fig. 6B). These findings suggested a mixed distribution of solid and cystic components. The interpretation of the imaging was that the patient exhibited a desmoplastic ameloblastoma.

A biopsy was performed with local anesthesia, resulting in the diagnosis of clear cell odontogenic carcinoma accompanied by ameloblastoma. Numerous clear cells were observed in tumor islands without an inconspicuous stellate reticulum (Fig. 7). The clear cells were periodic acid-Schiff positive and resistant to diastase digestion; however, the clear cells did not uptake alcian blue staining. Immunohistochemical examination was performed as follows: Formalin-fixed paraffin-embedded tissues were cut at $2 \mu \mathrm{m}$ thickness. Ki-67, $\mathrm{p} 53$, and $\mathrm{p} 63$ proteins were retrieved by autoclaving at $121^{\circ} \mathrm{C}$ for $15 \mathrm{~min}$ in retrieval buffer ( $0.1 \mathrm{M}$ citrate buffer; Mitsubishi Yatron, Tokyo, Japan). The S-100-protein was retrieved without autoclaving.

The sections were incubated with the following diluted primary antibodies: Rabbit anti-human S-100-protein polyclonal antibody (1:1; cat. no. 422091; Nichirei, Tokyo, Japan), mouse anti-human Ki-67 monoclonal antibody (1:100; cat. no. 00095324; M1B-1, Dako; Agilent Technologies, Inc., Santa Clara, CA, USA), mouse anti-human p53 monoclonal antibody (1:100; cat. no. 20023361; DO-7, Dako; Agilent Technologies, Inc.), and mouse anti-human p63 monoclonal antibody (1:25; cat. no. 147817; 7JUL, Novocastra; Leica Biosystems (Newcastle) Ltd., Newcastle, UK) for $30 \mathrm{~min}$ at

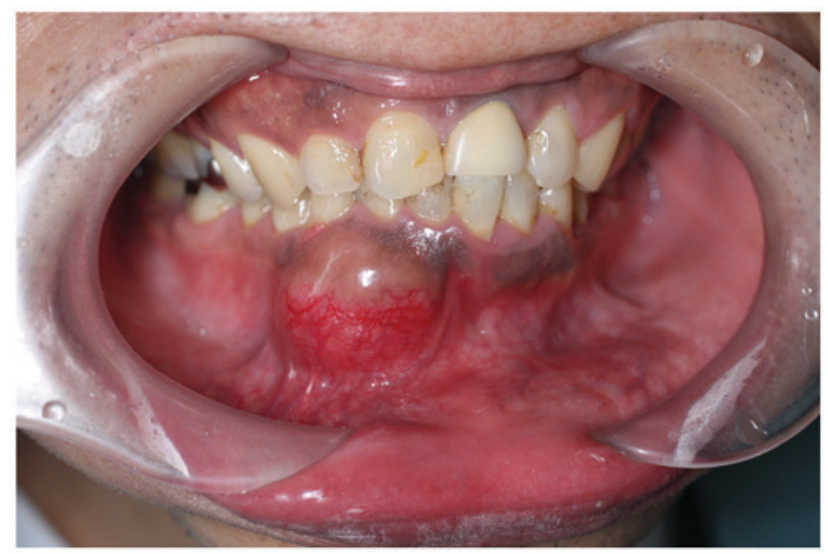

Figure 1. Intra-oral view, including an intra-oral swelling in the right canine area of the mandible. A localized, elastic hard swelling in the right buccal vestibule around teeth $42-44$ is observed.

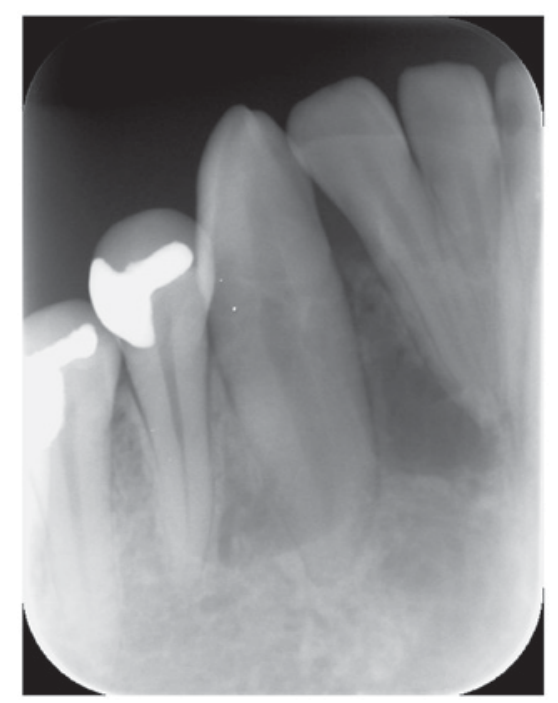

Figure 2. Intraoral x-ray of the tumor area. An initial intraoral x-ray image demonstrated a radiolucent lesion with delicate septa and margins in the nearby apexes of teeth $42-44$, mildly dislocating 42 and 43 .

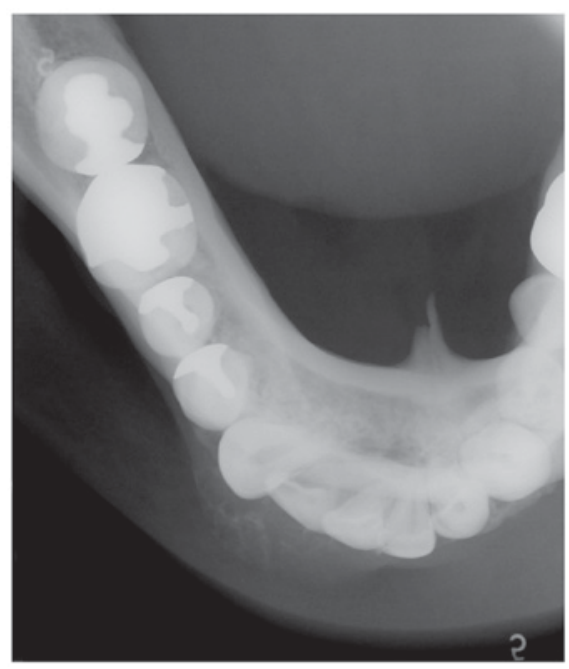

Figure 3. Occlusal x-ray image of the right side of the manible. An occlusal $\mathrm{x}$-ray image revealed a radiolucent-radiopaque lesion with a buccal bony expansion in the right lateral incisor and canine area of the mandible, illustrating a soap bubble-like appearance. 


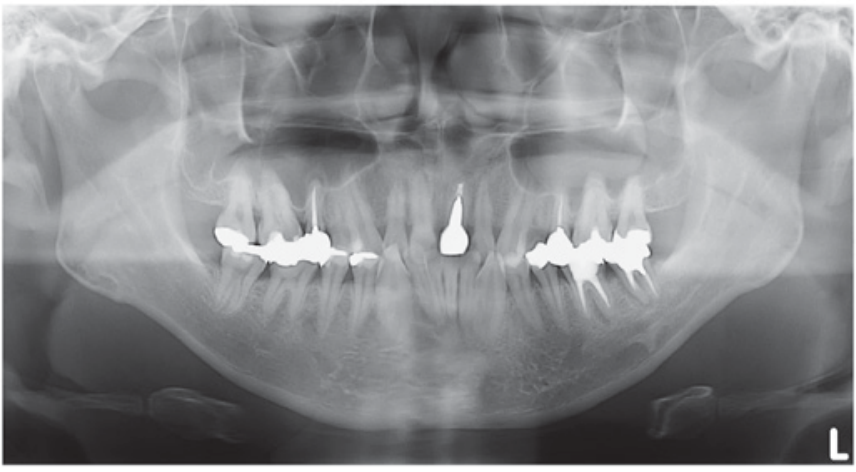

Figure 4. Panoramic x-ray image of the manible. No findings associated with the swelling were observed, and the image was affected by ghost images from the cervical vertebrae.



Figure 5. Computed tomography images of the mandible. (A) Axial view, (B) Higher axial view, (C) sagittal view. The images reveal an expanding, mixed radiolucent-radiopaque appearance in the area of teeth $42-44$, with irregularly thinned cortical plates.

A

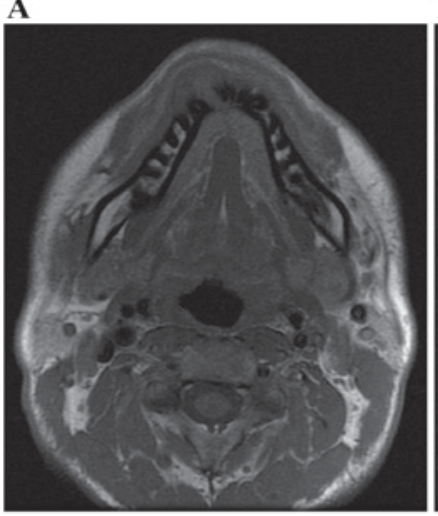

B

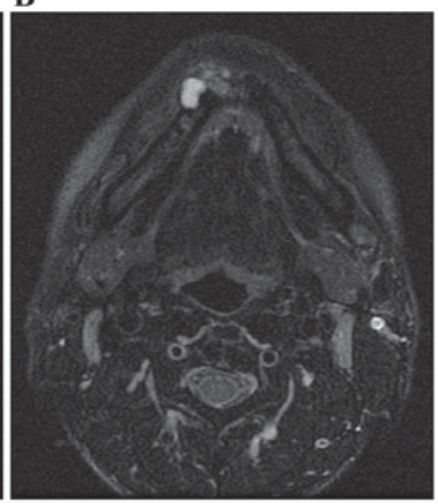

Figure 6. Magnetic resonance images. (A) T1-weighted image, demonstrating intermediate signal intensity around teeth 42-44. (B) T2-weighted fat-suppressed image, revealing variable intermediate and high signal intensity. These observations are indicative of a mixed pattern of solid and cystic components. room temperature. For the secondary antibody incubation, S-100-protein was incubated with peroxidase conjugated anti-rabbit antibody (1:1; cat. no. 10097631; Dako; Agilent Technologies, Inc.); Ki-67, p53, and p63 were incubated with a peroxidase conjugated anti-mouse antibody (1:1; cat. no. 10037259; Dako; Agilent Technologies, Inc.). The signals were then visualized using 3,3'-diaminobenzidine tetrahydrochloride and staining was considered to be positive if it was yellowish-brown. As negative control, normal mouse IgG and rabbit IgG were used instead of primary antibodies. This analysis revealed that the clear cells were negative for S-100-protein and Ki-67. p53-positive cells were infrequent, and p63 was expressed in the nuclei of the tumor cells.

With a tentative diagnosis of clear cell odontogenic carcinoma, the tumor was excised with a continuity of the lower border of the mandible (Fig. 8A). The remaining mandible was immediately reinforced with a mandibular reconstruction titanium plate (Fig. 8B). The postoperative course was uneventful; there has been no evidence of recurrence or metastatic disease for 2 years and 3 months subsequent to surgery.

Following the marginal resection of the mandible, histological examination of the tumor identified features inconsistent with those of the biopsy, including features of a typical plexiform-type ameloblastoma (Fig. 9). No clear cells were evident in the intraoperative biopsy or in a thorough examination of the entire specimen. Taking all the observations into account, the final diagnosis of CCAM was made. The patient provided informed consent for the publication of the present study, which was approved by the Ethics Review board of Osaka Dental University.

\section{Discussion}

The course of this patient indicated two notable aspects of ameloblastoma. Firstly, detailed CT and MRI findings of CCAM in the mandible of a patient were reported; to the best of our knowledge, these imaging characteristics were not previously described. Secondly, not until the histological examination of the surgical specimen could a definite diagnosis of CCAM be produced.

The imaging features closely resembled those of desmoplastic ameloblastoma. On intraoral and occlusal images, the lesion was radiolucent and multilocular, with a honeycombed or bubble-like appearance. The tumor expanded onto the cortex of the mandible, with subsequent tumor extension into the adjacent soft tissues that could be observed in the axial T1WI MR images. However, based purely on MR image findings, the osseous nature of the tissue would likely have remained undetected. In CT images, bony expansion with mildly scalloped marginal sclerosis, nonhomogeneous septum and displacement of the teeth were observed; these findings would typically be indicative of an ameloblastoma desmoplastic variant.

To the best of our knowledge, in previous literature, just 9 cases of CCAM have been reported, for which the imaging characteristics of intraoral or panoramic X-rays have been described as an ill-defined area of bone destruction and an extensive radiolucent lesion displacing the roots of the second premolar and the second molar, involving the floor of the left maxillary sinus as well as producing buccal expansion into 
A

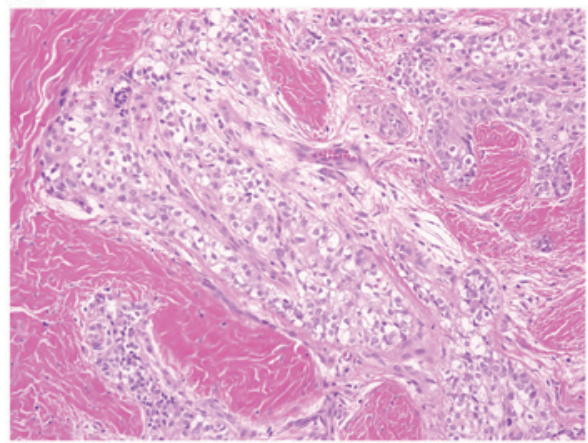

B

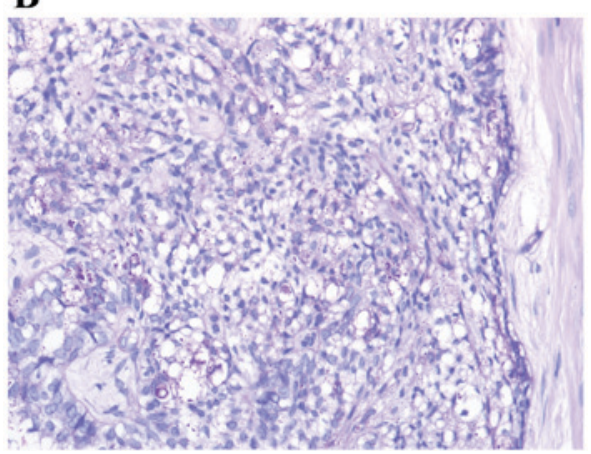

Figure 7. Immunohistochemical staining of the lesion following biopsy. (A) Numerous clear cells were observed in tumor islands without inconspicuous stellate reticulum. (B) Clear cells were stained positively by periodic acid-Schiff. Magnification, x100.

\section{A}

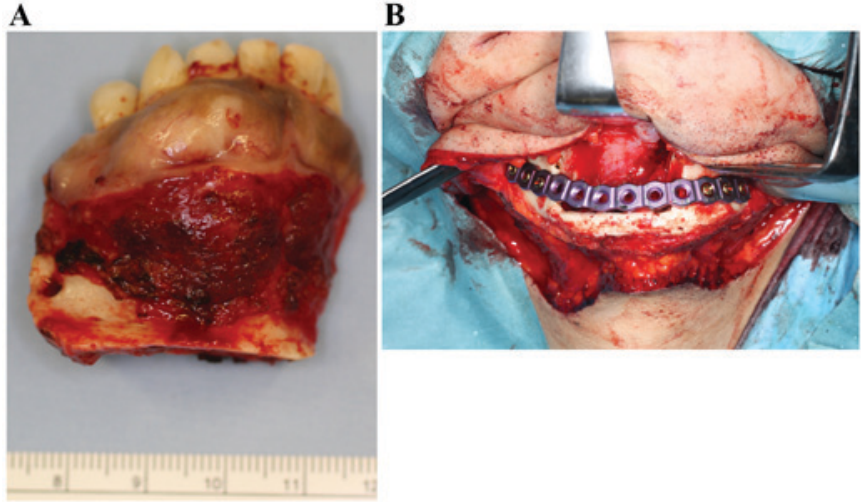

Figure 8. Photographs of the resected tumor and the mandible of the patient following surgery. (A) Excised specimen, identified as a clear cell ameloblastoma, with adjacent teeth and alveolar bone. (B) The surgical defect was immediately reconstructed with a titanium plate.

the vestibule (5), an ill-defined, multilocular radiolucency (3), an irregular bone destruction with truncation of the roots of related teeth (7), a poorly defined radiolucency with a regional resorption of the teeth (8) and a multilocular radiolucency (9). Only the study by Mari et al (4) described the CT findings from CCAM, and the images were limited to a recurrent tumor. In the first recurrence, a CT scan revealed a well-demarcated tumor that filled the maxillary sinus, affecting the nasal septum and floor of the right orbit. In the second recurrence, a CT scan showed a massive recurrence affecting the orbital cavity and the anterior cranial fossa (4). The present case exhibited a number of the previously described distinguishing

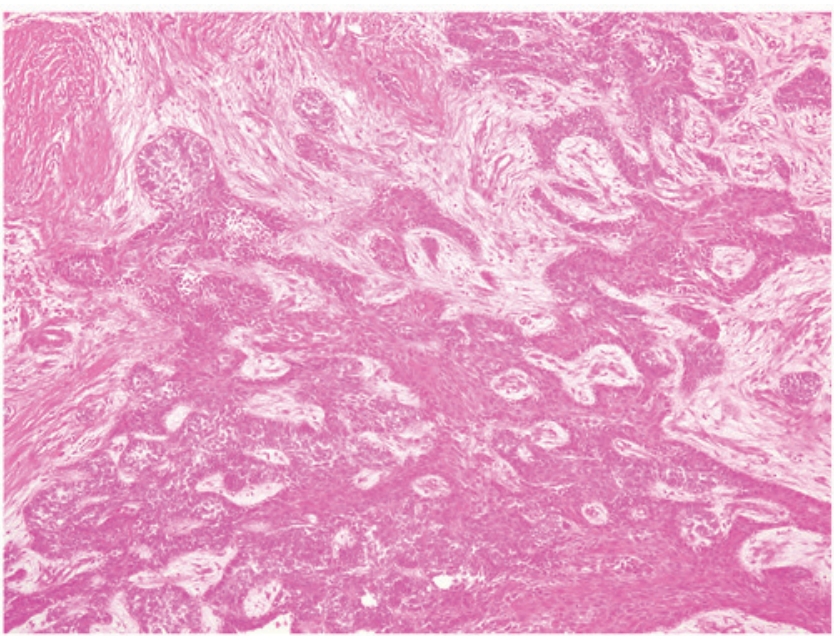

Figure 9. Histopathological photograph of the specimen obtained from the lesion following the resection. The specimen exhibited the features of a typical plexiform-type ameloblastoma. No clear cells were evident. Magnification, $\mathrm{x} 100$.

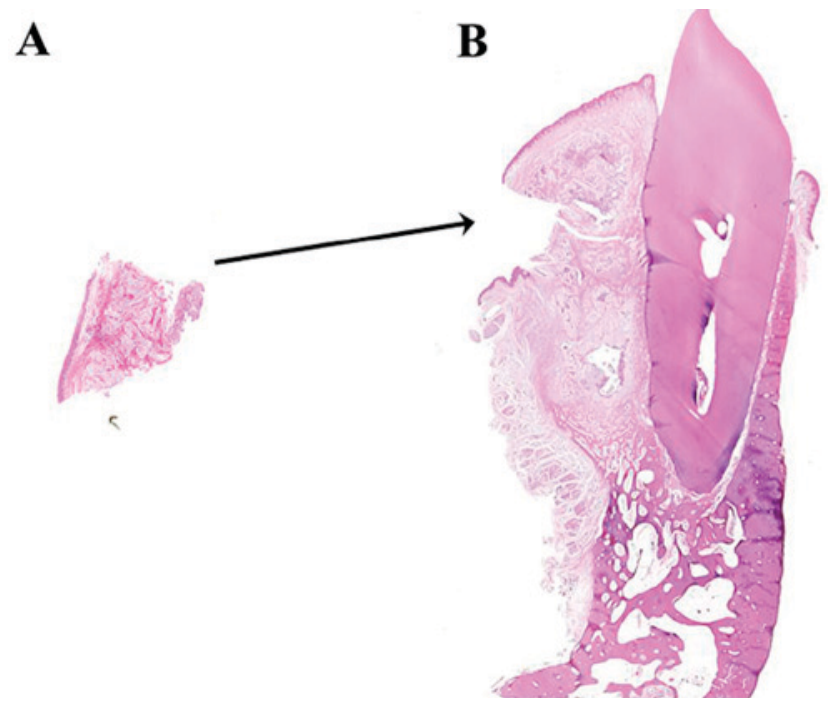

Figure 10. Images from the biopsy contrasted with images from the excised sample. (A) Clear-cell containing section of the biopsy. (B) Remaining section of the surgical specimen. These images are not magnified.

characteristics, as it was ill-defined with multilocular radiolucency and the displacement of the associated teeth. In particular, the intraoral image from the present case (Fig. 2) and Muller and Slootweg (3) bear a resemblance, in presenting a displacement of the teeth without resorption. Additionally, the CT images in this case confirmed that the tumor exhibited a locally invasive nature, consistent with the description by Mari et al (4).

It remains a matter of speculation why the histology results of the incisional biopsy and the surgical specimen were entirely different. One possibility is that sections prepared from the surgical specimen failed to contain any clear cells. The clear cells may have been lost between the incisional biopsy and the histologic examination of the surgical specimen; numerous clear cells were observed in sheets of epithelium in the former, whereas only solid hyperplasia of ameloblastic nests could be observed in the latter. A further possibility is that 
the biopsy-section (Fig. 10A) contained the entire clear cell component of the tumor. As a consequence, the remaining section prepared from the surgical specimen (Fig. 10B) did not include any further clear cells. If this was the case, an additional biopsy obtained from a different site should have been performed to offset the error. In either case, sampling error, the pitfall inherently existing in histopathological diagnosis, was likely a contributing factor. Based on the present case, performing a re-biopsy in a case with the histological finding of clear cells in addition to the CT/MRI findings of desmoplastic ameloblastoma may be advisable. Fig. 10A and $\mathrm{B}$ demonstrate a thought-provoking puzzle in the differences between the biopsy and surgical specimens. Neither the pathologist from the present study nor previous literature had ever reported this unusual situation.

As a result, the presence of clear cell elements presents a diagnostic challenge, and differentiating CCAM from similar entities may be enigmatic. However, the presence of clear cells in an ameloblastoma should be expected due to their origin from the dental lamina, which has been reported to contain clear cells (10). The proportion of clear cells in the lesion has varied in previous reports, from tumors that are composed almost entirely of clear cells $(9,11)$, to tumors containing a significant portion of other cellular elements $(3,10)$. When the other component is ameloblastic, the lesion has been described as CCAM $(5,7)$. In the present case, the neoplasm demonstrated a consistent biphasic histological pattern, with some areas resembling a plexiform-type ameloblastoma, and other areas resembling a clear cell component.

On immunohistochemical examination, the clear cells from the present case were negative for S-100-protein and Ki-67 expression. Antigens have been reported as negative (including vimentin, desmin, enolase, smooth muscle actin, calponin, S-100 protein, human melanoma black-45, $\alpha$ (1)-chymotrypsin, CD10, CD31, CD45, glial fibrillary acidic protein and chromogranin) or mildly positive in expression (keratin 13 and epithelial membrane antigen) for clear cell odontogenic carcinoma cells (11). A small number of previous reports, as reviewed by Loyola et al (11), have evaluated Ki-67 expression in clear cell carcinoma; low proliferative activity $(<8 \%)$ was exhibited by the tumors. However, it has been established that S-100 and Ki-67 expression may be negative in clear cell carcinoma and CCAM. Loyola et al (11) stated that 'a challenging scenario appears in the differential diagnosis of clear cell odontogenic carcinoma from ameloblastoma with clear cells'. The present case may also have good cause to misdiagnose CCAM as clear cell carcinoma.

In differential diagnosis, the clear cell odontogenic tumor described by Hansen et al should be considered (12); this tumor exhibited fibroblastic cellular stroma between the epithelial nests of clear cells. Ameloblastic differentiation, stellate reticulum and any resemblance to dental lamina were lacking (13). Other types of neoplasm, including the clear cell variant of calcifying epithelial odontogenic tumor, odontogenic fibroma, clear cell variant of mucoepidermoid carcinoma, clear cell squamous carcinoma and metastasis from renal carcinoma or hypernephroma should also be considered in the differential diagnosis $(12,13)$. It is always appropriate to rule out metastasizing disease when clear cell tumors of the jaw are encountered.

In conclusion, the present case study has illustrated the CT and MRI findings for CCAM in the mandible of a patient, including a characteristic example of the process of reaching a final diagnosis. This case should serve as a valuable warning that a definitive diagnosis for CCAM should be based on a combination of clinical and histopathological features.

\section{References}

1. Regezi JA, Kerr DA and Courtney RM: Odontogenic tumors: Analysis of 706 cases. J Oral Surg 36: 771-778, 1978.

2. Luna MA and Wenig BM: Polymorphous low-grade adenocarcinoma. In: World Health Organization Classification of Tumors. Pathology and Genetics Head and Neck Tumours. Barnes L, Eveson J, Reichart P and Sidransky D (eds.) IARC Press, Lyon, pp223-224, 2005.

3. Müller H and Slootweg P: Clear cell differentiation in an ameloblastoma. J Maxillofac Surg 14: 158-160, 1986.

4. Marí A, Escutia E, Carrera M and Pericot J: Clear cell ameloblastoma or odontogenic carcinoma. A case report. J Craniomaxillofac Surg 23: 387-390, 1995.

5. Waldron CA, Small IA and Silverman H: Clear cell ameloblastoma-an odontogenic carcinoma. J Oral Maxillofac Surg 43: 707-717, 1985.

6. Braunshtein E, Vered M, Taicher S and Buchner A: Clear cell odontogenic carcinoma and clear cell ameloblastoma: A single clinicopathologic entity? A new case and comparative analysis of the literature. J Oral Maxillofac Surg 61: 1004-1010, 2003.

7. Odukoya $\mathrm{O}$ and Arole O: Clear-cell ameloblastoma of the mandible (a case report). Int J Oral Maxillofac Surg 21: 358-359, 1992.

8. de Aguiar MC, Gomez RS, Silva EC and de Araújo VC: Clear-cell ameloblastoma (clear-cell odontogenic carcinoma): Report of a case. Oral Surg Oral Med Oral Pathol Oral Radiol Endod 81: 79-83, 1996.

9. Shamim T, Ipe Varghese V, Shameena PM and Sudha S: Clear cell ameloblastoma in a young child-an enigma in diagnosis. Indian J Pathol Microbiol 50: 362-364, 2007.

10. Wysocki GP, Brannon RB, Gardner DG and Sapp P: Histogenesis of the lateral periodontal cyst and the gingival cyst of the adult. Oral Surg Oral Med Oral Pathol 50: 327-334, 1980.

11. Loyola AM, Cardoso SV, de Faria PR, Servato JP, Barbosa de Paulo LF, Eisenberg AL, Dias FL, Gomes CC and Gomez RS: Clear cell odontogenic carcinoma: Report of 7 new cases and systematic review of the current knowledge. Oral Surg Oral Med Oral Pathol Oral Radiol 120: 483-496, 2015.

12. Hansen LS, Eversole LR, Green TL and Powell NB: Clear cell odontogenic tumor-a new histologic variant with aggressive potential. Head Neck Surg 8: 115-123, 1985.

13. Eversole LR: On the differential diagnosis of clear cell tumours of the head and neck. Eur J Cancer B Oral Oncol 29B: 173-179, 1993. 